\title{
Simultaneous Alignment and Structure Prediction of RNAs
}

\section{Are Three Input Sequences Better Than Two?}

\author{
Beeta Masoumi and Marcel Turcotte \\ School of Information Technology and Engineering, \\ University of Ottawa, \\ Ottawa, Ontario, Canada \\ \{bmasoumi, turcotte\}@site.uottawa.ca
}

\begin{abstract}
Comparative RNA sequence analyses have contributed remarkably accurate predictions. The recent determination of the $30 \mathrm{~S}$ and $50 \mathrm{~S}$ ribosomal subunits brought more supporting evidence. Several inference tools are combining free-energy minimisation and comparative analysis to improve the quality of secondary structure predictions. Using many input sequences should improve the accuracy, reduce the likelihood that bad predictions are made, but also lower the sensitivity. To investigate these claims, we have extended the software system Dynalign to use three input sequences, rather than two, and tested our algorithm with 10 tRNAs and $135 \mathrm{~S}$ rRNAs. The following hypotheses were tested: 1) the use of three input sequences improves the average accuracy compared to predictions based on two input sequences. Also, it should be less likely that all three input sequences simultaneously fold into a bad free-energy minimum compared to predictions based on two sequences, consequently, 2) the worse prediction (minimum accuracy) for any sequence should be more accurate when three input sequences are used rather than two. Finally, the consensus structure of three sequences is probably less representative of the individual sequences. 3) Therefore, the average coverage should be less.
\end{abstract}

\section{Introduction}

The repertoire of known non-protein coding RNAs (ncRNAs) is growing rapidly 1]. The housekeeping roles of RNAs, such as those of the tRNA, rRNA, RNAseP, snRNA and snoRNA, were established early. In the recent years, it has become clear that RNAs also have important regulatory functions. Examples include microRNAs, which regulate the expression of protein genes by targeting a complementary region of their mRNAs. MicroRNAs constitute one of the most abundant class of regulatory molecules, and are key to many developmental processes [2. Several discoveries collectively demonstrate that untranslated messenger RNAs can sense the level of metabolites, and modulate the expression of certain genes accordingly. Those RNAs are referred to as RNA sensors and riboswitches [3, 4]. Post-transcriptional regulation of gene expression often involves 
secondary structure elements located in the untranslated regions of mRNAs 5 . Consequently, detailed knowledge of RNA secondary and tertiary structure is essential to help understand RNA functions.

RNA secondary structure prediction methods have been thoroughly evaluated. In particular, Gardner and Giegerich have performed a comprehensive evaluation of comparative RNA structure prediction methods [6]. Doshi et al. reviewed specifically free-energy minimisation methods that are using the nearestneighbour model[7]. One of their main conclusions is that free-energy minimisation methods based on the nearest-neighbour model work best for shorter sequences, such as tRNA or $5 \mathrm{~S}$ rRNA, for which they reported an average accuracy for predictions of $69 \%$ and $71 \%$ respectively.

Recently, Mathews and Turner developed, and published, a software system combining free-energy minimisation and comparative sequence analysis for finding the minimum free-energy structure common to two input sequences 8 . The computer system, called Dynalign, greatly improves the accuracy of secondary structure predictions compared to free-energy minimisation alone.

Herein, we extend this algorithm to use three input sequences, rather than two, and investigate the performance of the new computer program. We called this software system eXtended-Dynalign, or X-Dynalign for short, to emphasise its origin.

This paper is organised as follows. Section 2 outlines the algorithm. In Section [3 the main hypotheses to be empirically evaluated are laid out, the datasets are described, and the evaluation measures are defined. Section 4 presents the results. Section 5 concludes and discusses the results.

\section{Algorithm}

Dynalign is a pragmatic implementation of the algorithm proposed by Sankoff for solving simultaneously the RNA folding and alignment problems 9 . Dynalign is restricted to two input sequences, while the original proposal was formulated for an arbitrary set of $N$ input sequences. Also, Dynalign introduces a constraint on the maximum distance between aligned nucleotides so as to reduce the execution time. This is analogous to the banding technique that is used for sequence alignment.

$\mathrm{X}$-Dynalign is a direct extension of Dynalign. It takes as input three sequences and produces a three-way sequence alignment as well as a common secondary structure. The objective function consists of a linear combination of the freeenergy of each sequence, given the common secondary structure, and an empirical term for gap penalties.

$$
\Delta G_{\text {total }}^{\circ}=\Delta G_{\text {sequence 1 }}^{\circ}+\Delta G_{\text {sequence 2 }}^{\circ}+\Delta G_{\text {sequence 3 }}^{\circ}+\Delta G_{\text {gaps }}^{\circ}
$$

where $\Delta G_{\text {sequence } i}^{\circ}$, for $i \in\{1,2,3\}$, represents the conformational free-energy of the sequence $i$ when folded onto the common secondary structure, according to the nearest-neighbour model. 
Three sets of recurrence equations are defining the objective function: $W, V$ and $W 9$. Equations of the form $W(i, j, k, l, m, n)$ represent the minimum freeenergy for the optimal alignment and structure prediction of $S_{1}[i . . j], S_{2}[k . . l]$ and $S_{3}[m . . n]$, when $i, k$ and $m$ are aligned, and $j, l$ and $n$ are also aligned, where $S_{i}$ denotes the sequence $i$ and $S_{i}[a . . b]$ represents the fragment of $S_{i}$ comprising the nucleotides $a, a+1 \ldots b$. Equations of the form $V(i, j, k, l, m, n)$ represent the minimum free-energy assuming that $i$ and $j, k$ and $l$, and $m$ and $n$ are simultaneously aligned but also base-paired. Finally, $W 9(i, k, m)$ represents the minimum free-energy for the prefix alignment of $S_{1}[1 . . i], S_{2}[1 . . k]$ and $S_{3}[1 . . m]$. A detailed description of the recurrence equations can be found in [10. The recurrence equations are solved using dynamic programming. The algorithm requires $\mathcal{O}\left(\left|S_{1}\right|^{2} M^{4}\right)$ space and $\mathcal{O}\left(\left|S_{1}\right|^{3} M^{6}\right)$ time, where $M$ is a constant that limits the maximum distance between aligned nucleotides.

\section{Methodology}

\subsection{Experiments}

The following hypotheses are tested: 1) the use of three input sequences should improve the average accuracy compared to predictions based on two input sequences. When three input sequences are used, the likelihood that they all three fold into a bad free-energy minimum should be less than when two input sequences are used, consequently, 2) the worse prediction (minimum accuracy) should be more accurate when three input sequences are used rather than two. Finally, the secondary structure common to three input sequences should be less representative of the individual sequences, consequently, 3) the average coverage should be less. But first, we determine empirically the optimum gap penalties for these datasets.

\section{$3.2 \quad$ Datasets}

Input sequences were selected such that they can be aligned optimally with a small value of $M$. Obviously, this information would not be known in advance in most cases. Also, the input sequences were filtered so that the maximum pairwise identity was less than $90 \%$. A total of 10 tRNA sequences from the original paper were used. Their pairwise sequence identity varies from 27.3 to $68.8 \%$. The secondary structure assignments were taken from the compilation by Sprinzl et al. 11,12. A set of $135 \mathrm{~S}$ rRNA sequences was built using information obtained from the Comparative RNA Web Site 13, 14, 15. Their pairwise sequence identity varies from 47.2 to $88.2 \%$.

\subsection{Performance Measures}

We call references, the secondary structures that were obtained from the tRNA compilation by Sprinzl and the Comparative RNA Web Site. We define as true 
positives (TP) the base pairs that are occurring in both structures, reference and predicted, false positives $(\mathrm{FP})$, the base pairs that are occurring in the predicted structure but not in the reference one, and false negatives $(\mathrm{FN})$, the base pairs that are occurring in the reference structure but not in the predicted one. Offsets were not allowed.

The positive predictive value (sometimes called PPV, specificity or accuracy) is defined as the fraction of the predicted base pairs that are also present in the reference structure, $\mathrm{TP} /(\mathrm{TP}+\mathrm{FP})$. The sensitivity (coverage) is defined as the fraction of the base pairs from the reference structure that are correctly predicted, TP/(TP + FN $)$. Finally, we also measured the Matthews Correlation Coefficient, as defined by Gorodkin, Stricklin and Stormo[16]:

$$
\sqrt{\frac{\mathrm{TP}}{(\mathrm{TP}+\mathrm{FN})} \times \frac{\mathrm{TP}}{(\mathrm{TP}+\mathrm{FP})}}
$$

\section{Results}

\subsection{Calibrating Gap Penalties}

In [8], the optimal gap penalty was found to depend on the class of RNA; 2.0 and $0.4 \mathrm{Kcal} / \mathrm{mol}$ for the tRNA and $5 \mathrm{~S}$ rRNA, respectively. Accordingly, we performed two sets of experiments to measure the effect of various gap penalty scores on PPV, sensitivity and MCC. Since these experiments are time consuming, only six gap penalty scores were tested, $0.0,0.25,0.5,1.0,2.0,4.0$, and only triples that can be aligned with a small value of $M$, here 5 , were selected. In all, 105 and 90 predictions were made for the tRNA and 5S rRNA, respectively. For the experiments presented herein, we have chosen a gap penalty score of 1.0 $\mathrm{Kcal} / \mathrm{mol}$, because it corresponds to the maximum sensitivity for both datasets, tRNA and 5S rRNA.

\subsection{Comparative Analysis}

We present the analysis of the tRNA data first. Nine runs, 27 predictions, were made using X-Dynalign, while 19 runs, 38 predictions, were made using Dynalign. The mean PPV, sensitivity and MCC are 96.8 $\pm 7.6,94.4 \pm 7.5$ and $95.6 \pm 7.3$ for X-Dynalign, and $92.1 \pm 14.6,89.1 \pm 15.7$ and $90.5 \pm 15.0$ for Dynalign. Our data represent a subset of that of Mathews and Turner, the PPV for Dynalign measured on this subset is 5.7 percentage points higher than theirs. We observe that the use of three sequences improves all three indices and reduces their variance, for this particular dataset.

Table 1 presents the performance indices per sequence. Dynalign performed well in the best case scenario. For all the sequences, it was possible to find a pair of input sequences having a high positive predictive value. The maximum PPV for every entry is 100, except for that of RS0380. Further analysis shows that the structure of RS0380 (tRNA ${ }^{\text {Asp }}$ Haloferax volcanii) has an extra stem 
Table 1. PPV for the tRNA dataset. The subscripts $x d$ and $d$ are designating XDynalign and Dynalign respectively. $N$ is the number of predictions

\begin{tabular}{lrrrrrrrr}
\hline Id & $\mathrm{N}_{x d} \mathrm{~N}_{d} \mathrm{Min}_{x d} \mathrm{Min}_{d} \mathrm{Max}_{x d} \mathrm{Max}_{d}$ & $\mathrm{Ave}_{x d}$ & $\mathrm{Ave}_{d}$ \\
\hline $\mathrm{RD} 0260$ & 4 & 5 & 100 & 80 & 100 & 100 & 100.0 & 96.0 \\
$\mathrm{RD} 0500$ & 4 & 5 & 76 & 45 & 100 & 100 & 82.2 & 80.8 \\
$\mathrm{RD} 4800$ & 5 & 5 & 100 & 80 & 100 & 100 & 100.0 & 96.0 \\
$\mathrm{RE} 140$ & 2 & 4 & 100 & 100 & 100 & 100 & 100.0 & 100.0 \\
$\mathrm{RE} 6781$ & 2 & 4 & 100 & 77 & 100 & 100 & 100.0 & 94.3 \\
$\mathrm{RF} 6320$ & 4 & 5 & 95 & 45 & 100 & 100 & 96.4 & 89.1 \\
$\mathrm{RL} 0503$ & 1 & 2 & 100 & 100 & 100 & 100 & 100.0 & 100.0 \\
$\mathrm{RL} 1141$ & 2 & 3 & 100 & 70 & 100 & 100 & 100.0 & 90.3 \\
$\mathrm{RS} 0380$ & 1 & 2 & 100 & 83 & 100 & 87 & 100.0 & 85.2 \\
$\mathrm{RS} 1141$ & 2 & 3 & 100 & 70 & 100 & 100 & 100.0 & 90.3 \\
\hline
\end{tabular}

in the variable loop, that X-Dynalign predicted more accurately. For 9 out of 10 experiments, the maximum sensitivity for X-Dynalign equals or exceeds that of Dynalign.

Both algorithms are seeking to find a structure that minimises a linear combination of the free-energy of each input sequence given the common structure. Using three input sequences should have a positive impact on the worse case scenario. It should be less likely that all three input sequences jointly fold into the wrong minimum free-energy structure than with two input sequences. Our data support this observation, for all the entries the minimum PPV for X-Dynalign is the same or better than that of Dynalign. For 8 out of 10 sequences, the minimum PPV is 100, in one case, the minimum PPV is 95, and for one case the minimum PPV is 76 . The two sequences leading to the worse predictions are RD0500 and RF6320, see Figure 1. Dynalign produces an elongated structure.

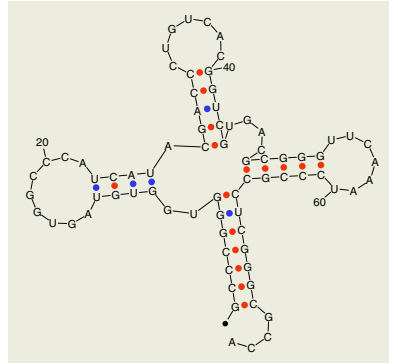

(a)

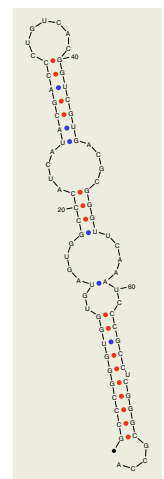

(b)

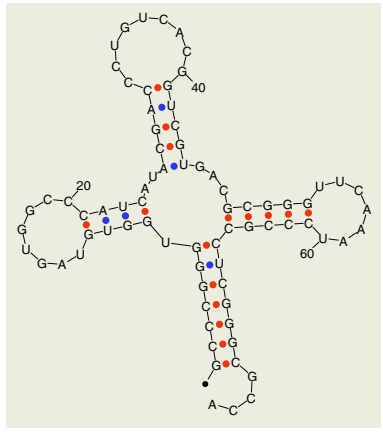

(c)

Fig. 1. Reference (a), Dynalign (b) and X-Dynalign (c) secondary structures for the tRNA RD0500 
Table 2. PPV for the $5 \mathrm{~S}$ dataset

\begin{tabular}{lrrrrrrrr}
\hline $\mathrm{Id}$ & $\mathrm{N}_{x d} \mathrm{~N}_{d} \mathrm{Min}_{x d} \mathrm{Min}_{d} \mathrm{Max}_{x d} \mathrm{Max}_{d}$ & $\mathrm{Ave}_{x d}$ & $\mathrm{Ave}_{d}$ \\
\hline $\mathrm{AJ} 131594$ & 2 & 3 & 100 & 91 & 100 & 100 & 100.0 & 94.5 \\
$\mathrm{AJ} 251080$ & 6 & 5 & 88 & 82 & 90 & 86 & 90.3 & 84.8 \\
$\mathrm{D} 11460$ & 6 & 5 & 87 & 66 & 87 & 88 & 87.6 & 79.4 \\
$\mathrm{~K} 02682$ & 8 & 9 & 63 & 88 & 100 & 97 & 89.1 & 92.0 \\
$\mathrm{M} 10816$ & 3 & 4 & 90 & 85 & 90 & 88 & 90.7 & 87.8 \\
$\mathrm{M} 16532$ & 1 & 2 & 94 & 77 & 94 & 85 & 94.1 & 81.8 \\
$\mathrm{M} 25591$ & 6 & 5 & 87 & 82 & 90 & 86 & 89.8 & 84.8 \\
$\mathrm{~V} 00336$ & 3 & 4 & 75 & 65 & 100 & 100 & 91.9 & 91.4 \\
$\mathrm{X} 02024$ & 9 & 6 & 88 & 82 & 90 & 88 & 90.1 & 85.8 \\
$\mathrm{X} 02627$ & 1 & 2 & 100 & 92 & 100 & 100 & 100.0 & 96.0 \\
$\mathrm{X} 04585$ & 2 & 3 & 72 & 68 & 94 & 93 & 83.4 & 82.7 \\
$\mathrm{X} 08000$ & 5 & 5 & 90 & 88 & 90 & 90 & 90.6 & 89.4 \\
$\mathrm{X} 08002$ & 5 & 5 & 90 & 88 & 90 & 90 & 90.6 & 89.4 \\
\hline
\end{tabular}

However, using a third sequence increases the accuracy by more than 30 percentage points. The structure produced by X-Dynalign has the overall cloverleaf shape, however, the nucleotides of the first part and second part of the D-arm are shifted by one and two positions, respectively. The minimum coverage is generally good. For all the sequences the coverage is $75 \%$ or better. For all the tests the coverage for X-Dynalign is the same as Dynalign or better.

For our second test set, we have 19 runs, 57 predictions, using X-Dynalign, and 29 runs, 58 predictions, using Dynalign. The mean PPV, sensitivity and MCC are $90.3 \pm 5.8,76.6 \pm 5.3$ and $83.2 \pm 5.5$ for X-Dynalign, and $87.7 \pm 7.4,79.2 \pm$ 6.7 and $83.3 \pm 6.7$ for Dynalign. For this particular dataset, the performance of both systems is comparable on the basis of the Matthews correlation coefficient. What is gained in accuracy is lost in sensitivity.

Table 2 presents the performance indices per sequence. Using three input sequences improves the worse (PPV) prediction for 12 out of 13 sequences. Also, for 10 out of 13 sequences, the minimum PPV obtained is $85 \%$ or more. The minimum sensitivity is the same or improved for 11 out of 13 sequences. However, the maximum sensitivity exceeds that of Dynalign for 2 out of 13 sequences.

The prediction of the 5S rRNA of Micrococcus lutus (K02682) has an accuracy of $63 \%$ only. We believe this is due to the fact that single base pair insertion has not been implemented yet in X-Dynalign. In the triple K02682, V00336 and X04585, the structure of Rhodobacter capsulatus (X04585) has a shorter helix IV, 7 base pairs compared to 8 for the other two structures.

\section{Conclusion and Discussion}

We have extended the software system Dynalign to use three input sequences, rather than two. The resulting system is called eXtended-Dynalign (X-Dynalign for short). Its time/space complexity limits its application to 1 ) short sequences (say less than 
$200 \mathrm{nt}$ ) and 2) sequences that can be aligned optimally with a small value of $M$ (less than 6 ), where $M$ is the maximum distance of aligned positions.

The strengths of Dynalign carry over to the new system. Namely, it improves the accuracy of secondary structure predictions compared to predictions based on a single input sequence. It requires no sequence homology.

It also shares some of its limitations. In particular, the gap penalties are treated as a separate term in the objective function. The optimal value has to be determined empirically. In [8], it was found that the optimal value for this term depends on the class of RNA studied. In our limited experiments, the dependency seems less important. It also seems that there is large plateau were several gap penalty scores are leading to a nearly optimal solution; w.r.t. PPV, for example. Our key conclusions are:

- The lowest PPV for any prediction is generally improved when using three input sequences;

- The average accuracy is improved;

- The average sensitivity of the algorithm slightly degraded for the 5S rRNA dataset. However, a per sequence analysis shows that the majority of the lowest sensitivity scores are higher for X-Dyanlign than Dynalign;

- X-Dynalign is able to reproduce subtle details, such as the prediction of a stem in the variable region of certain tRNAs.

There are several obvious directions for extending this class of algorithms, such as handling pseudo-knots and reporting suboptimal structures. However, one of the most urgent improvement is to reduce the time/space complexity. Several runs presented herein take up to week to compute on some of the fastest processors available today.

The detailed knowledge of RNA secondary structure is essential for understanding the sequence-structure-function relationships. X-Dynalign takes advantage of the paramount of data that is accumulating in sequence databases. Because it requires no sequence homology, X-Dynalign should be useful to comparative RNA sequence analyses.

\section{Availability}

The source code, written in $\mathrm{C}++$, as well as the scripts for calculating the performance indices are made available under the GNU General Public Licence from http://bio.site.uottawa.ca/software/x-dynalign. Supplementary material, including additional tables and figures, can be found on our web site.

\section{References}

1. Storz, G.: An expanding universe of noncoding RNAs. Science 296 (2002) 12601263

2. Bartel, D.P.: MicroRNAs: Genomics, biogenesis, mechanism, and function. Cell 116 (2004) 281-297 
3. Nudler, E., Mironov, A.X.: The riboswitch control of bacterial metabolism. Trends Biol. Sci. 29 (2004) 11-17

4. Lai, E.C.: RNA sensors and riboswitches: Self-regulating messages. Current Biology 13 (2003) R285-R291

5. Mignoe, F., Gissi, C., Liuni, S., Pesole, G.: Untranslated regions of mRNAs. Genome Biology 3 (2003) 0004.1-0004.10

6. Gardner, P.P., Robert, G.: A comprehensive comparison of comparative RNA structure prediction approaches. BMC Bioinformatics 5 (2004) 140

7. Doshi, K.J., Cannone, J.J., Cobaugh, C.W., Gutell, R.R.: Evaluation of the suitability of free-energy minimization using mearest-neighbor energy parameters for rna secondary structure prediction. BMC Bioinformatics 5 (2004) 105

8. Mathews, D., Turner, D.: Dynalign: An algorithm for finding the secondary structure common to two RNA sequences. J. Mol. Biol. 317 (2002) 191-203

9. Sankoff, D.: Simultaneous solution of RNA folding, alignment and protosequence problems. SIAM J. Appl. Math. 45 (1985) 810-825

10. Masoumi, B.: A dynamic programming algorithm for the simultaneous alignment and structure prediction of three RNA sequences. Master's thesis, School of Information Technology and Engineering, Faculty of Engineering, University of Ottawa (2005)

11. Sprinzl, M., Horn, C., Brown, M., Ioudovitch, A., Steinberg, S.: Compilation of tRNA sequences and sequences of tRNA genes. Nucl. Acids Res. 26 (1998) 148-153

12. Sprinzl, M., Vassilenko, K.S.: Compilation of tRNA sequences and sequences of tRNA genes. http://www.uni-bayreuth.de/departments/biochemie/trna (2003)

13. Gutell, R.R.: Comparative RNA web site. http://www.rna.icmb.utexas.edu (2004)

14. Cannone, J.J., Subramanian, S., Schnare, M.N., Collett, J.R., D’Souza, L.M., Du, Y., Feng, B., Lin, N., Madabusi, L.V., Muller, K.M., Pande, N., Shang, Z., Yu, N., Gutell, R.R.: The comparative RNA web (CRW) site: An online database of comparative sequence and structure information for ribosomal, intron, and other RNAs. BMC Bioinformatics 3 (2002)

15. Cannone, J.J., Subramanian, S., Schnare, M.N., Collett, J.R., D’Souza, L.M., Du, Y., Feng, B., Lin, N., Madabusi, L.V., Muller, K.M., Pande, N., Shang, Z., Yu, N., Gutell, R.R.: The comparative RNA web (CRW) site: An online database of comparative sequence and structure information for ribosomal, intron, and other RNAs: Corrections. BMC Bioinformatics 3 (2002)

16. Gorodkin, J., Stricklin, S.L., Stormo, G.D.: Discovering common stem-loop motifs in unaligned RNA sequences. Nucl. Acids Res. 29 (2001) 2135-2144 